\title{
L'ecocardiografia e il nefrologo: una "pocket guide" per nefrologi curiosi
}

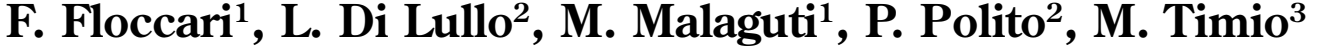 \\ ${ }^{1}$ UOC di Nefrologia e Dialisi, Ospedale San Paolo, Civitavecchia (Roma) \\ ${ }^{2}$ UOC di Nefrologia e Dialisi, Ospedale San Giovanni Evangelista, Tivoli (Roma) \\ ${ }^{3}$ Dipartimento di Nefrologia e Dialisi, Ospedale San Giovanni Battista, Foligno (PG)
}

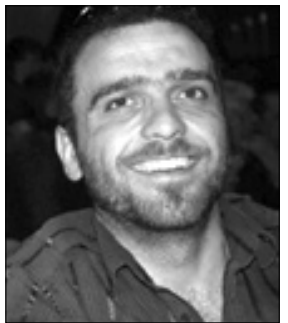

\section{Introduzione}

La semeiotica medica, tra la fine del Novecento e il Millennio in corso, è stata letteralmente rifondata dalla diffusione in corsia delle tecniche ecografiche più disparate. L'esame obiettivo, che nei millenni ha conservato e affinato i gesti dei padri della Scienza Medica, sempre più

Fulvio Floccari

oggi comprende una vera e propria appendice ecografica, eseguita in tempo reale direttamente dal medico clinico, in misura proporzionata alle conoscenze e alla formazione del medico stesso.

L'esame obiettivo "nefrologico" può avere diverse finalità: dalla valutazione del peso secco e delle cause di dispnea nel paziente in dialisi, alla diagnosi differenziale tra insufficienza renale acuta o cronica, ostruttiva o meno ecc... A ciascuna di queste finalità l'esame ecografico di base conferisce sensibilità e specificità, abbrevia l'iter diagnostico, riduce i costi e limita il numero di esami richiesti, abbreviando di conseguenza le liste di attesa agli stessi.

Ad esempio anche il nefrologo meno esperto, acquisiti i primi rudimenti tecnici ecografici, può verificare la presenza di dilatazione delle vie urinarie in corso di insufficienza renale acuta. Ciò abbrevierà in maniera virtuosa l'iter diagnostico terapeutico, accelererà il ricorso all'urologo o ad altro collega che si sia dedicato specificamente alla diagnostica ecografica, limitando i costi della diagnosi e fornendo una risposta terapeutica pronta e mirata.

L'ecografia addominale e vascolare è stata a tal punto implementata nella pratica clinica nefrologica quotidia- na, che essa è ormai compresa tra le competenze nefrologiche di base da un'ampia percentuale di colleghi. Vi sono però organi che anche il nefrologo "ecografista" è restio a valutare: cuore, polmoni, aorta ecc... Eppure la ricerca delle "comete" all'esame ecografico del torace, la valutazione seriata di versamenti pericardici, pleurici o addominali sono solo alcuni degli esempi tangibili delle informazioni preziose, disponibili al nefrologo che abbia il coraggio di spostare la sonda di pochi gradi da luoghi a lui ormai familiari.

L'avventura del nefrologo in ultrasonografia può trovare oggi nuove opportunità di crescita, approcciando anche all'ecocardiografia.

Tale tecnica, una volta ritenuta di esclusiva pertinenza cardiologica, è oggi entrata di diritto nella semeiotica ecografica di numerosi altri specialisti. Chi frequenta corsi di base di ecocardiografia osserverà, tra i discenti, anestesisti, cardiochirurghi, internisti, medici dell'urgenza e, appunto, nefrologi. Il futuro di ogni specialità dipende infatti grandemente dalla capacità di vincere la naturale obsolescenza tecnica delle metodiche, rifondandole e innovandole, allargando il proprio punto di vista all'organo che più di ogni altro dialoga costantemente col rene: il cuore.

Un ecocardiogramma color Doppler "di base" è una valida metodica di screening della popolazione a rischio, affidabile sia come strumento diagnostico che prognostico in tutti gli ambiti della patologia cardiovascolare: ipertensione arteriosa, insufficienza cardiaca, valvulopatie, malattie del pericardio. Si tratta poi di una metodica non invasiva, ripetibile e poco costosa.

Un numero così elevato di lati positivi ne ha ovviamente accresciuto la domanda, troppo spesso non accompagnata da una proporzionale crescita nell'offerta, così da 
allungare anche notevolmente le liste di attesa all'esame. La corretta indicazione a un ecocardiogramma, lì dove origini dalla clinica, comporta invece che l'esame venga svolto in tempi brevi, ancor di più nel paziente uremico, le cui condizioni emodinamiche sono ciclicamente soggette a brusche variazioni.

$\mathrm{E}$ allora forse giunto il momento che il nefrologo "medio" accetti l'invito a interessarsi anche di ecocardiografia, per acquisire ciò che la metodica può aggiungere al suo carnet di strumenti culturali, pur limitandosi ad applicazioni nefrologiche della stessa. In altri termini può il nefrologo effettuare un primo screening dei suoi pazienti, salvo poi chiedere al cardiologo di visionare coloro che risultino portatori di patologia di rilievo? O ancora: possiamo seguire nel tempo, in maniera seriata, un paziente che il cardiologo ci ha segnalato portatore di patologia? In tal caso molte misure della geometria cardiaca, semplici e ripetibili, si presterebbero a una analisi costante e cadenzata, basti pensare ad esempio alla volumetria atriale o agli spessori di parete.

Approcciandosi ai primi rudimenti di ecocardiografia il nefrologo può trovare inoltre una miniera di stimoli culturali, capaci di mutarne l'atteggiamento clinico. La pratica ecocardiografica fornisce infatti non solo le risposte a molteplici quesiti clinici, ma anche le giuste domande da porre al collega cardiologo, che rimane il vero e unico padrone della materia specialistica. Il dialogo, la contaminazione tra due specialità così vicine, la frequentazione che nasce dal condividere almeno in parte la metodica ecocardiografica, possono aiutare cardiologi e nefrologi a incrociare le conoscenze, nell'interesse ultimo del paziente.

Questa sintetica guida all'ecocardiografia offrirà quindi al nefrologo una rapida descrizione della semeiotica ecocardiografica, puntando sugli aspetti di maggiore utilità alla pratica nefrologica. Lungi dalla convinzione di fornire in maniera così sintetica una panoramica completa della metodica, il nostro obiettivo sarà innanzitutto incuriosire i colleghi più coraggiosi e attrarli all'ecografia.

\section{L'ecocardiografo}

L'ecocardiografia di primo livello non necessita di ecocardiografi "dedicati", sicuramente molto costosi e difficilmente reperibili al nefrologo medio.

Un buon esame di screening può avvalersi infatti di comuni monitor ecografici, purché provvisti di "seconda armonica tissutale" o "harmonic fusion", ossia capaci di garantire una buona definizione di immagine, minimizzando la generazione di artefatti. Uno studio valvolare completo non può poi prescindere dalla disponibilità del modulo Doppler continuo e di una sonda cardiologica. Questa è una sonda micro-convessa, dotata cioè di un sottile fascio ultrasonico convesso, sufficientemente "agile" da attraversare le esili finestre acustiche parasternali. La frequenza di emissione/ricezione della sonda dovrà essere compresa tra 2 e 7,5 MHz.

Il software cardiologico, necessario a raccogliere le misure ecocardiografiche, è di norma residente nel software di base dell'apparecchio. Il costo dell'implementazione tecnologica di un ecografo "normale", purché non sia decisamente vetusto, si aggira pertanto (tra sonda e Doppler continuo) attorno ai settemila euro (la cifra è indicativa e riferita all'adeguamento di un GE pro-Logic 7 o di un comunissimo Logic 3 ).

$\mathrm{Nel}$ caso invece il nefrologo decida di non interessarsi affatto dello studio valvolare, l'apparecchio non necessiterà del Doppler continuo, pressocché dimezzando i costi di aggiornamento. L'ecografo "base", dotato della sonda micronvex sarà comunque in grado di eseguire un esame morfologico completo, che comprenda la misurazione dell'area atriale o degli spessori di parete, la misura della frazione di eiezione, la ricerca di versamenti pericardici, la valutazione della funzione diastolica, lo studio della morfologia e della dinamica degli apparati valvolari.

\section{L'esame}

La valutazione ecocardiografica completa comporta l'utilizzo completo di tutte le risorse tecnologiche dell'ecografo:

M - Mode

B - Mode

Color Doppler

Doppler pulsato

Doppler continuo.

Uno studio specialistico cardiologico può giovarsi anche del Doppler Tissutale, presente unicamente su apparecchiature dedicate, ma certamente non necessario al nefrologo interessato a un esame di base.

Per una corretta esecuzione dell'esame il paziente viene posizionato sul lettino ambulatoriale in decubito laterale sinistro rivolto verso l'operatore con il braccio sinistro piegato e posizionato sotto il capo e il braccio destro posizionato disteso lungo il fianco omolaterale (Fig. 1).

Il monitor è posizionato dal lato opposto rispetto al capo del paziente e l'operatore siede di fronte al monitor con il paziente alla sua destra, mentre la sonda è posizionata nella mano destra. Tale posizione, oltre a ovvie ragioni di ergonomia e comodità dell'operatore, consente al paziente di ottenere informazioni su un esame che spesso 


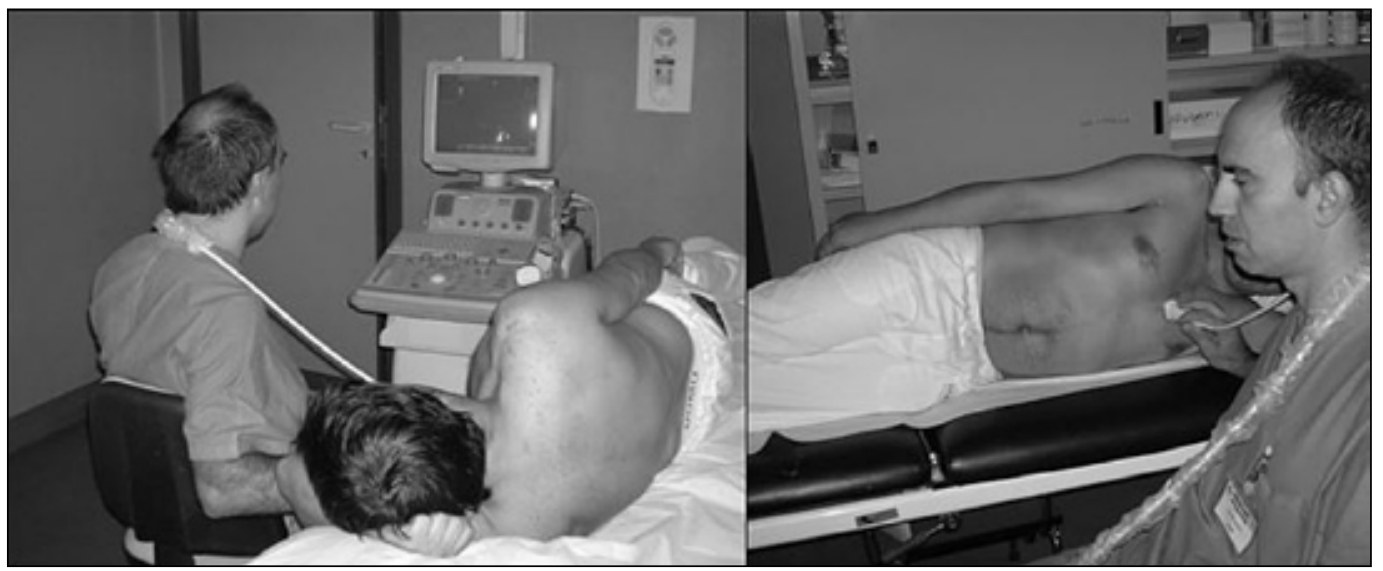

Fig. 1 - Corretto posizionamento del paziente.

incute apprensione, permettendo così all'utente di viverlo con maggior serenità e meglio tollerare un esame la cui durata può anche essere superiore ai trenta minuti. Le proiezioni ecocardiografiche di base sono 4 (Fig. 2):

Parasternale asse lungo

Parasternale asse corto

Apicale 4 camere

Apicale 5 camere.

L'esame può essere completato con altre 2 proiezioni complementari:

Sottocostale

Soprasternale (Notch).

\section{Proiezione parasternale asse lungo}

Tale proiezione si ottiene posizionando il trasduttore all'altezza del terzo-quarto spazio intercostale sulla linea parasternale sinistra, con il repere della sonda rivolto verso il fianco destro del paziente. Una buona proiezione si ottiene poi aggiustando la posizione della sonda con piccoli movimenti di traslazione e inclinando di pochi gradi il fascio di ultrasuoni. È bene avere chiaro che non esiste un unico repere corretto e ideale per tutti i pazienti e che solo con pazienza ed esercizio si arriva a sviluppare la giusta confidenza con questa operazione. L'immagine ottenuta rappresenta una sezione ideale condotta lungo l'asse lungo del ventricolo sinistro e ci consente di visualizzare la radice aortica e l'atrio sinistro. Sempre in questa proiezione, spostandosi sempre lungo l'asse lungo del muscolo cardiaco, è possibile valutare i diametri del ventricolo destro e del ventricolo sinistro (VS) e lo spessore del setto interventricolare (SIV) e della parete posteriore del VS (PPVS) (Fig. 3). L'analisi, che inizia con uno studio B-mode della cinetica e della morfologia, si completa con le misurazioni M-Mode dei singoli diametri e spessori, misurati da

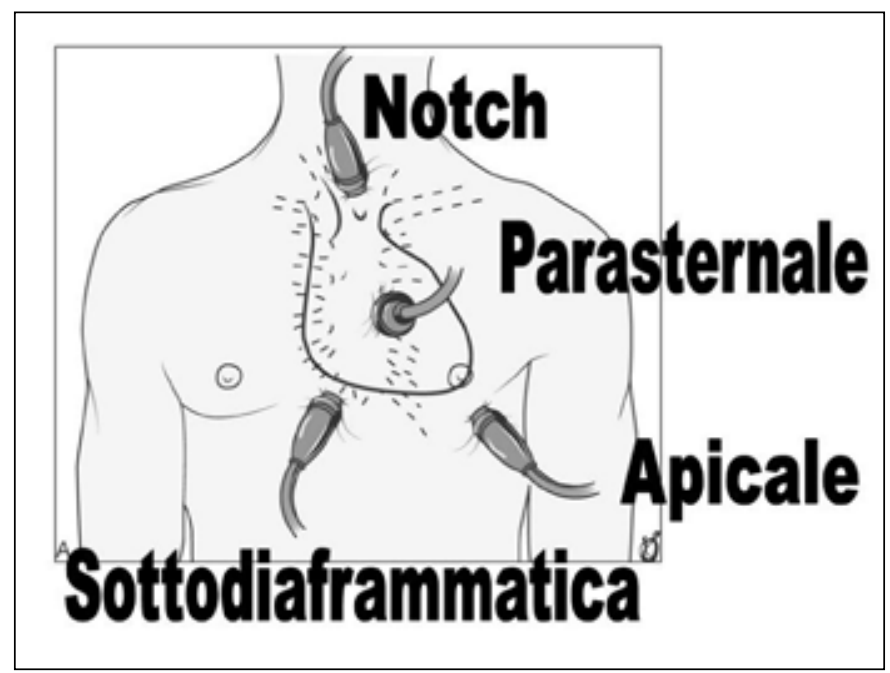

Fig. 2 - Proiezioni ecocardiografiche di base.

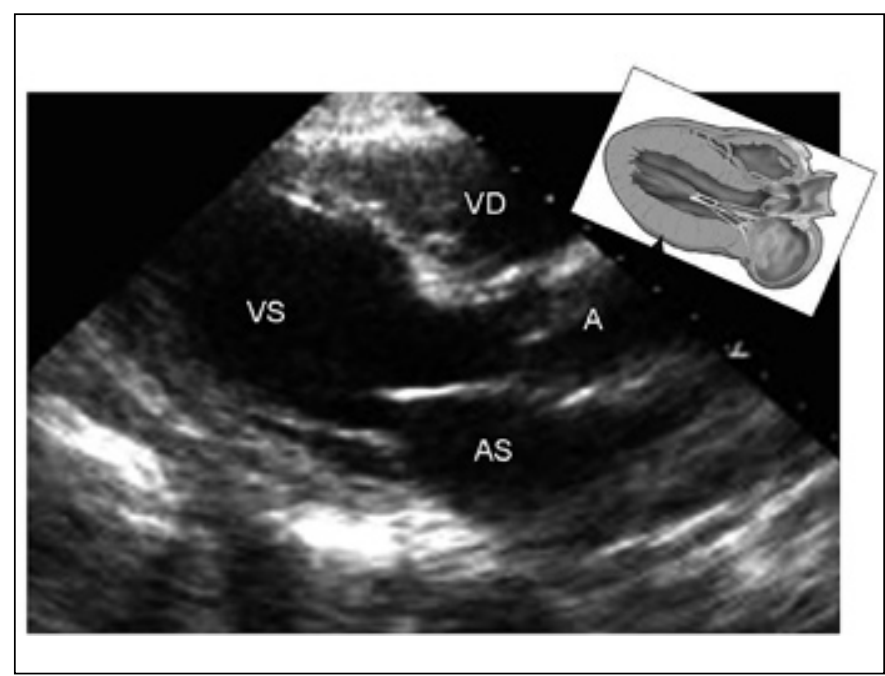

Fig. 3 - Proiezione parasternale asse lungo. (VS=Ventricolo sinistro, $\mathrm{VD}=$ Ventricolo destro, $\mathrm{A}=$ Aorta, $\mathrm{AS}=$ =Atrio sinistro). 
bordo endocardico a bordo endocardico.

I diametri del VS e gli spessori del SIV e della PPVS vengono misurati sia in sistole che in diastole, mentre i diametri del ventricolo destro (VD) vengono misurati unicamente in fase tele-diastolica.

La misurazione degli spessori del SIV, del VS e della PPVS con la conseguente valutazione dell'indice di massa cardiaca permettono di differenziare tra un'ipertrofia concentrica (senza dilatazione ventricolare) e una eccentrica (con dilatazione) del VS.

In tale proiezione è inoltre possibile visualizzare la valvola mitrale, la cuspide aortica destra e la non coronarica, e il tratto d'efflusso del ventricolo sinistro (Left Ventricle Outflow Tract, LVOT) con i primi centimetri di vaso aortico, che ben si prestano alla misurazione dei diametri.

Potremmo cioè da qui avere già contezza di un sospetto aneurisma della radice aortica, eventualmente da studiare in maniera ulteriore da proiezione Notch.

Da questa proiezione inizia poi lo studio delle valvole aortica e mitralica. In presenza di stenosi aortica o mitralica i rispettivi lembi e cuspidi appariranno ispessiti e iperecogeni e caratterizzati da scarsa escursione tra sistole e diastole.

La presenza di jet di rigurgito, che descriveremo più avanti, sui due apparati valvolari compresi nella proiezione, sarà indicativo di insufficienze valvolari meritevoli di approfondimento nelle successive proiezioni.

\section{Proiezione parastenale asse-corto}

La proiezione asse corto si ottiene ruotando in senso orario il trasduttore, a partire dalle proiezione asse lungo, di un angolo compreso tra 45 e $90^{\circ}$, in maniera tale da ottenere una proiezione ortogonale rispetto alla precedente. Le immagini proiettate sembrano osservate dal basso, cioè dall'apice cardiaco verso la base.

Scorrendo dall'alto verso il basso, con questa proiezione possiamo ottenere la visualizzazione dell'apice del ventricolo sinistro, dei muscoli papillari, della valvola mitrale, della valvola aortica e della biforcazione del tronco polmonare.

Questa proiezione è di grande utilità, non solo per misurare le dimensioni dell'area valvolare mitralica e aortica, ma soprattutto perché consente un'analisi della cinetica ventricolare sinistra "a tutto tondo". Particolarmente al nefrologo che si avvicina per le prime volte all'ecocardiografia, tale proiezione rende immediatamente distinguibili le varie porzioni del VS (pare-

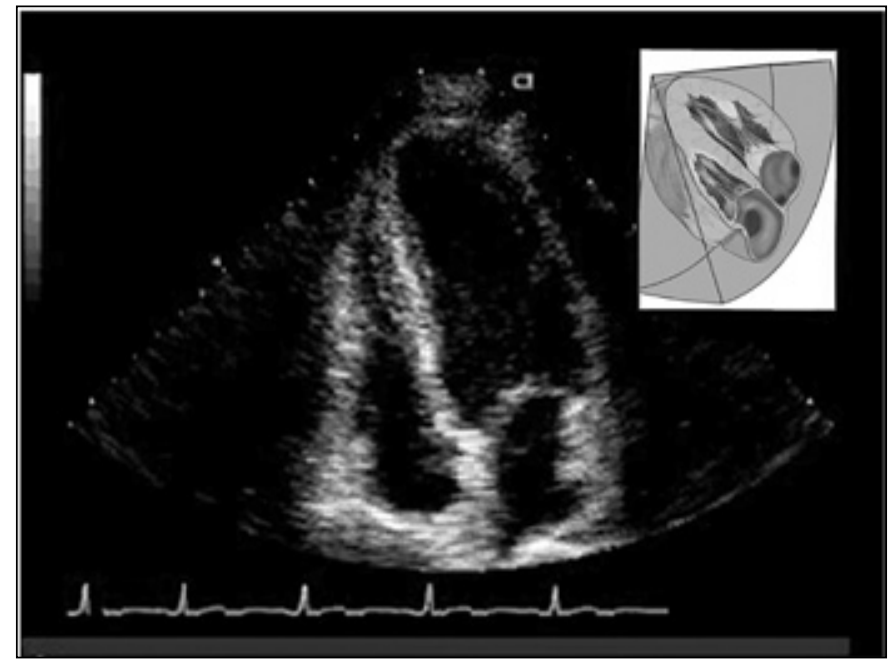

Fig. 4 - Proiezione apicale quattro camere.

te anteriore, laterale, posteriore ecc.), presentandole all'operatore in una chiave estremamente intuitiva, che consente di aggirare la difficoltà iniziale del neofita nell'attribuire a ogni segmento osservato la giusta nomenclatura.

\section{Proiezione apicale 4 camere}

La proiezione apicale 4 camere si ottiene ponendo il trasduttore a livello dell'apice cardiaco dopo aver individuato l'itto della punta. In questo modo risultano individuabili le 4 camere cardiache, il SIV e il setto interatriale (SIA), la valvola mitrale e la valvola tricuspide (Fig. 4).

Rispetto allo schermo potremmo avere le sezioni sinistre sulla destra o sulla sinistra del nostro monitor (e le sezioni destre dalla parte opposta) a seconda di come è posizionato il repere della sonda.

Si tratta senza dubbio della proiezione che per definizione risulta gradita al neofita, che vi può facilmente riconoscere le principali strutture cardiache, essendo tra tutte quella maggiormente intuitiva. In questa proiezione è possibile effettuare la misurazione dell'area delle camere atriali, lo studio della cinetica ventricolare sinistra, delle valvole mitrale, tricuspide e polmonare, la ricerca di eventuali versamenti pericardici. Si tratta pertanto della proiezione di maggiore utilità "nefrologica", consentendo di verificare la presenza di dilatazione atriale (un segno precoce di sovraccarico di volume), di calcificazioni valvolari o dell'anello mitralico, di versamenti pericardici o grossolane alterazioni della cinetica ventricolare. 


\section{Proiezione apicale 5 camere}

È una proiezione estremamente simile alla precedente, che si ottiene partendo appunto dalla proiezione 4 camere e inclinando leggermente in alto e in avanti il trasduttore. Questo movimento consente la visualizzazione della radice e dei lembi valvolari aortici (Fig. 5). È la misurazione standard per lo studio della valvola aortica, consentendo il miglior allineamento possibile con il vaso.

\section{Proiezione sottocostale}

In alcuni pazienti le precedenti proiezioni non consentono di esplorare in maniera soddisfacente le diverse sezioni cardiache; è il caso, ad esempio, dei pazienti affetti da BPCO che presentano un'elevata impedenza acustica toracica. Il trasduttore viene posizionato al centro rispetto alla linea sottocostale e permette di visualizzare (se inclinato verso il basso) il parenchima epatico, le vene epatiche e una sezione asse-corto della vena cava inferiore. Inclinando il trasduttore verso l'alto si identificano gli sbocchi delle vene epatiche nella vena cava inferiore. Con un opportuno gioco di inclinazione della sonda, tale proiezione consente lo studio di tutte le strutture cardiache, risultando comoda per completare uno studio reso difficoltoso da finestre parasternali esigue. Tale proiezione è accessibile anche con una comune sonda convessa da ecografia addominale, seppure con le ovvie limitazioni dovute ad artefatti strumentali.

\section{Proiezione soprasternale (Notch)}

Si ottiene posizionando la testa del trasduttore a livello della fossa soprasternale con il suo asse lungo sul bordo sinistro della trachea. In tal modo diventano visualizzabili l'aorta ascendente, l'arco dell'aorta e l'aorta toracica discendente.

Questa proiezione diventa di fondamentale aiuto per la misurazione dei diametri dell'arco aortico e dell'aorta ascendente e per il conseguente studio di eventuali dilatazioni aneurismatiche dell'aorta.

\section{L'esame Doppler}

Come per altri organi e tessuti, le misurazioni Doppler in ecocardiografia si basano sull'effetto Doppler, il quale stabilisce che la frequenza di un suono aumenta a mano a mano che la sorgente sonora si avvicina all'osservato-

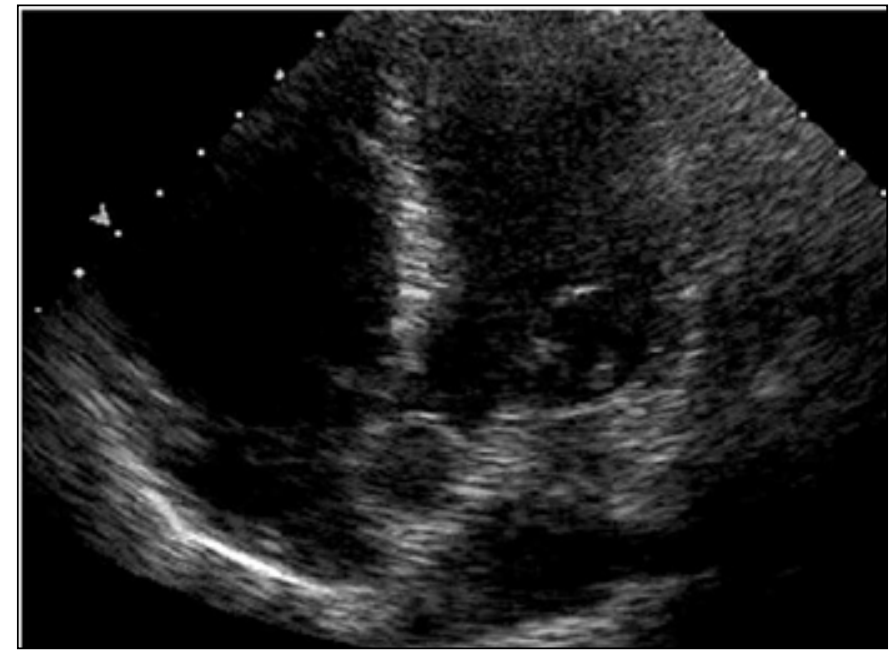

Fig. 5 - Proiezione apicale cinque camere.

re e diminuisce quando se ne allontana. La modificazione della frequenza fra suono trasmesso e suono riflesso è definita Variazione Doppler e dipende dalla frequenza trasmessa, dalla velocità di movimento del bersaglio (il sangue, nel nostro caso) e dall'angolo tra direzione del fascio e direzione del movimento delle emazie.

In ambito ecocardiografico il Doppler viene impiegato sia in modalità pulsata che continua e le due modalità sono assolutamente complementari. Nel Doppler PULSATO un singolo cristallo del trasduttore invia e riceve i fasci di ultrasuoni emettendo una breve sequenza di impulsi a una determinata frequenza (PRF - pulse repetition frequency); il Doppler pulsato misura le velocità di flusso in un punto preciso all'interno del volume campione. È di grande utilità pratica in quanto, durante il suo utilizzo, non si perde la possibilità di mantenere la visione in B mode delle strutture cardiache.

Nel Doppler CONTINUO invece ciò non avviene, in quanto il trasduttore è impegnato nell'emettere gli ultrasuoni e ricevere quelli riflessi, il tutto in maniera continua. Con questa modalità vengono misurate tutte le variazioni di frequenza incontrate dal fascio ultrasonoro durante il percorso, ma si perde la possibilità di "vedere" in $\mathrm{B}$ mode ciò che si sta puntando. La metodica continua presenta indubbiamente una maggiore affidabilità nella misurazione di velocità superiore ai $60-70 \mathrm{~cm} / \mathrm{sec}$ ed è quindi da utilizzarsi per lo studio della velocimetria aortica e mitralica.

Il COLOR DOPPLER si basa sui principi del Doppler pulsato e la codificazione del flusso avviene secondo le combinazioni di tre colori (rosso, verde e blu) sulla base di tre parametri: velocità, direzione ed estensione del flusso. 
Il flusso ematico diretto verso il trasduttore ha una variazione di frequenza positiva (la frequenza del suono riflesso è più alta di quella del suono trasmesso) ed è codificato in rosso, mentre il flusso che si allontana dal trasduttore è codificato in blu (variazione di frequenza negativa).

Il colore dei flussi diventa di importanza fondamentale nella valutazione dei rigurgiti transvalvolari. Variando la frequenza di emissione delle pulsazioni (PRF) si può scegliere di impostare un livello soglia di velocità al di sopra del quale effettuare la registrazione. Una corretta impostazione della PRF è di fondamentale importanza per avere una buona discriminazione di eventuali insufficienze valvolari.

Il Doppler TISSUTALE (TDI) viene invece utilizzato per valutare la velocità di movimento delle pareti cardiache, velocità inferiori a quelle del sangue in movimento. In corso di utilizzo di tale funzione, un filtro rigetta automaticamente le velocità medie e alte, registrando invece le basse velocità di movimento delle pareti cardiache.

Trattandosi di una tecnologia di interesse prevalentemente cardiologico, il TDI è implementato unicamente su ecocardiografi di fascia medio-elevata.

\section{Conclusioni}

Chiudiamo qui la prima parte della nostra rapida presentazione della metodica. Nel prossimo numero del Giornale avremo modo di presentare le applicazioni pratiche di quanto qui descritto. Saranno pertanto oggetto di discussione le tecniche di misurazione della performance sistolica e diastolica, oltre alle metodiche di valutazione delle valvulopatie e dei versamenti pericardici.

Indirizzo degli Autori:

Fulvio Floccari, MD

Via Monte Gennaro 30

00010 San Polo dei Cavalieri, Roma

fulviofloccari@gmail.com 